\title{
Protective Role of Human Intravenous Immunoglobulin from Influenza A Virus Infection in Mice
}

\author{
Katsuro Hagiwara ${ }^{1, *}$, Sachiyo Kawami ${ }^{1}$, Yuuko Kato-Mori ${ }^{1}$, Ritsuko Kubota-Koketsu ${ }^{2,5}$, \\ Muneo Tsujikawa ${ }^{3}$, Takeru Urayama ${ }^{2,3}$, Mikihiro Yunoki ${ }^{1,2,3, \#, *}$, Kazuo Takahashi ${ }^{4}$ and \\ Kazuyoshi Ikuta ${ }^{2}$ \\ ${ }^{I}$ School of Veterinary Medicine, Rakuno Gakuen University, Hokkaido, Japan \\ ${ }^{2}$ Department of Virology, Research Institute for Microbial Diseases, Osaka University, Osaka, Japan \\ ${ }^{3}$ Osaka Research Laboratory, Benesis Corporation, Osaka, Japan \\ ${ }^{4}$ Department of Infectious Diseases, Osaka Prefectural Institute of Public Health, Osaka, Japan \\ ${ }^{5}$ Kanonji Institute, The Research Foundation For Microbial Diseases of Osaka University, Kagawa, Japan
}

\begin{abstract}
Intravenous immunoglobulin (IVIG) has been manufactured from pooled plasma of 10,000 or more units from healthy donors. Recently, we reported that the IVIG manufactured even before the 2009 influenza pandemic contained antibodies reactive to seasonal H1N1 and pandemic H1N1 2009 (H1N1 pdm) viruses. In this study, we used an animal model to evaluate the efficacy of IVIG against influenza infections. A seasonal influenza H1N1 strain (New Caledonia, $\mathrm{A} / \mathrm{NC} / 20 / 99)$ and an H1N1 pdm strain (A/Osaka/168/2009) were used. The BALB/c and severe combined immunodeficiency mice (SCID; C.B-17/lcr-scid/scid) were also used. Mice inoculated with A/NC/20/99 or A/Osaka/168/2009 were administrated IVIG and monitored for 3 weeks. The administration of IVIG $48 \mathrm{~h}$ before and after inoculation with a mouse-adapted seasonal H1N1 virus, resulted in survival rates of 80 and $88 \%$, respectively. The rate among control mice was $30 \%$. In addition, infectivity in lungs from IVIG-treated mice also decreased significantly. Similar effects of IVIG on the survival rate were obtained with H1N1 pdm. Thus, IVIG was shown to be effective against both viruses in mice.
\end{abstract}

Keywords: IVIG, Influenza, 2009 Pandemic Influenza, Animal model.

\section{INTRODUCTION}

The influenza virus is currently the most important public health concern in the world, especially since the appearance of the 2009 pandemic influenza A/H1N1 (H1N1 pdm) virus. Human intravenous immunoglobulin (IVIG), a product manufactured from plasma derived from more than 10,000 units from healthy donors, most of whom have had natural infections with seasonal influenza viruses as well as vaccinations, could contain a wide variety of antibodies effective for protection against influenza infections.

In Japan, IVIG has not been yet approved for influenza virus infections, although it was recommended for influenza encephalopathy by the Study Group for Influenza Encephalopathy [1] and the Japanese Respiratory Society [2].

The mechanism of its effect against influenza is not yet clear. However, Yunoki et al. [3] and Hong et al. [4] reported that IVIG contains significant titers of hemagglutination-

\footnotetext{
*Address correspondence to these authors at the School of Veterinary Medicine, Rakuno Gakuen University. 582 Midorimachi, Bunkyodai, Ebetsu, Hokkaido 069-8501, Japan; Tel: +81-11-388-4826; Fax: +81-11-387-5890; E-mail: k-hagi@rakuno.ac.jp and Pathogenic Risk Management, Benesis Corporation. 2-6-18, Kitahama, Chuo-ku, Osaka, Osaka 541-8505, Japan; Tel: +81-6-6201-1679; Fax: +81-6-6231-4191;

E-mail: yunoki.mikihiro@mt-pharma.co.jp

"Current address of M. Yunoki: Pathogenic Risk Management, Benesis Corp
}

inhibition (HI) and viral neutralization (VN or $\mathrm{MN}$ ) antibodies against not only seasonal H1N1 but also H1N1 $\mathrm{pdm}$. The cross-reacting antibody against $\mathrm{H} 1 \mathrm{~N} 1 \mathrm{pdm}$ seemed to be derived from natural influenza viral infections as well as vaccinations. Here, we examined the efficacy of IVIG against these influenza infections in mice.

\section{MATERIALS AND METHODS}

Viruses

A seasonal influenza H1N1 strain (New Caledonia, $\mathrm{A} / \mathrm{NC} / 20 / 99)$ and an H1N1 pdm strain (A/Osaka/168/2009) were used. Before the experiment, the H1N1 and H1N1 pdm strains were passaged 5 times in BALB/c mice and 4 times in the severe combined immunodeficiency mice (SCID; C.B-17/lcr-scid/scid, CLEA Japan Inc., Japan). The mouseadopted viruses were named mo-A/NC/20/99 and moA/Osaka/168/2009, respectively. To evaluate the adaptation, $\mathrm{BALB} / \mathrm{c}$ mice (4-week-old males) were infected intranasally with 100 or 1000 focus-forming units (FFUs) of mo$\mathrm{A} / \mathrm{NC} / 20 / 99(\mathrm{n}=10)$ or mo-A/Osaka/168/2009 $(\mathrm{n}=7)$, then observed for 3 weeks.

\section{Human Intravenous Immunoglobulin (IVIG)}

The IVIG (Venoglobulin- $\mathrm{IH}^{\circledR}$; Benesis Corporation, Japan) used was the same lot previously show to contain 
1:160 $\mathrm{HI}$ and 1:640 VN titers against seasonal H1N1 (A/NC/ 20/99) and 1:8 $\mathrm{HI}$ and 1:64 VN titers against H1N1 pdm (A/ Osaka/168/2009) [3].

\section{Infection with mo-A/NC/20/99}

A total of $40 \mathrm{BALB} / \mathrm{c}$ mice (4-week-old males) were divided into four groups ( $\mathrm{n}=10$ per group). The mice were infected intranasally with mo-A/NC/20/99 at 1000 FFUs per head for the evaluation of IVIG's effect on survival for 3 weeks. To investigate the efficacy of IVIG treatment at $48 \mathrm{~h}$ before inoculation (hbi) or at $48 \mathrm{~h}$ post inoculation (hpi), each group of mice were intraperitoneally administered with IVIG at $5 \mathrm{mg}$ per head (corresponding to $250 \mathrm{mg} / \mathrm{kg}$ ) at 48 hbi, and at 48 and 72 hpi with 1000 FFUs per head. As a control, the H1N1-inoculated $\mathrm{BALB} / \mathrm{c}$ mice were orally administered $100 \mathrm{mg} / \mathrm{kg}$ of Oseltamivir (Tamiflu ${ }^{\circledR}$, Chugai Pharmaceutical Co., Ltd., Japan) for 4 days, and observed for 3 weeks. The dissected lung was fixed with $10 \%$ formalin$\mathrm{PB}$ and prepared for tissue sectioning and HE stained for histological examination.

\section{Infection with mo-A/Osaka/168/2009}

A total of $14 \mathrm{BALB} / \mathrm{c}$ mice were inoculated intranasally with mo-A/Osaka/168/2009 at $1000 \mathrm{FFUs}$ per head. The mice were divided into two groups for the evaluation of IVIG's effect on survival for 2 weeks. To evaluate the antiviral effect of IVIG on the H1N1 pdm virus in SCID mice lacking immunoglobulin and functional $\mathrm{T}$ and $\mathrm{B}$ cells, SCID mice ( 8 weeks old, male) were inoculated intranasally with mo-A/Osaka/168/2009 at 1000 FFUs. The mice were intraperitoneally administered IVIG at $5 \mathrm{mg}$ per head (corresponding to $250 \mathrm{mg} / \mathrm{kg}$ ) at $48 \mathrm{hpi}$. The untreated control group was administered the solvent of IVIG.

\section{Evaluation of Antiviral Effects}

Survival rates were monitored for 3 weeks. The BALB/c mice inoculated with mo-A/NC/20/99 or mo-A/Osaka/168/ 2009 were monitored. The infected SCID mice treated with or without IVIG were dissected at 3 and 5 days post inoculation (dpi). The lung from infected mice was homogenized with PBS (20\% homogenate) and infectivity $\left(\mathrm{TCID}_{50}\right)$ in the lung was determined on days 3 and 5 post administration of IVIG.

We used the commercial statistical analysis software (SPSS 15.0 J, SPSS Japan Inc., Tokyo, Japan) for all statistical analyses in this study. Independent t-test was used for comparisons of averages for two groups between IVIG treatment and untreatment control.

\section{RESULTS}

\section{Viral Adaptation to the Mice}

The BALB/c mice inoculated with the seasonal H1N1 strain at 100 and 1000 FFUs per head were observed for 3 weeks (data not shown). The survival rate was $30 \%$ among the mice infected with 1000 FFUs and $100 \%$ among those infected with 100 FFUs. In contrast, the survival rate of $\mathrm{BALB} / \mathrm{c}$ mice infected with H1N1 pdm at $1000 \mathrm{FFUs}$ was $33 \%$. The histological analysis of lungs from both BALB/c and SCID mice showed hemorrhagic pneumonia. HE-stained sections of dissected lung tissue from BALB/c mice infected with 1000 FFUs of mo-A/NC/20/99 are shown in Fig. (1). The titer of virus hereafter was 1000 FFUs per head.

\section{Efficacy Against H1N1 and H1N1 pdm}

As shown in Fig. (2A), the treatment of BALB/c mice with IVIG at 48 hpi with seasonal H1N1 was highly

\section{2 dpi}
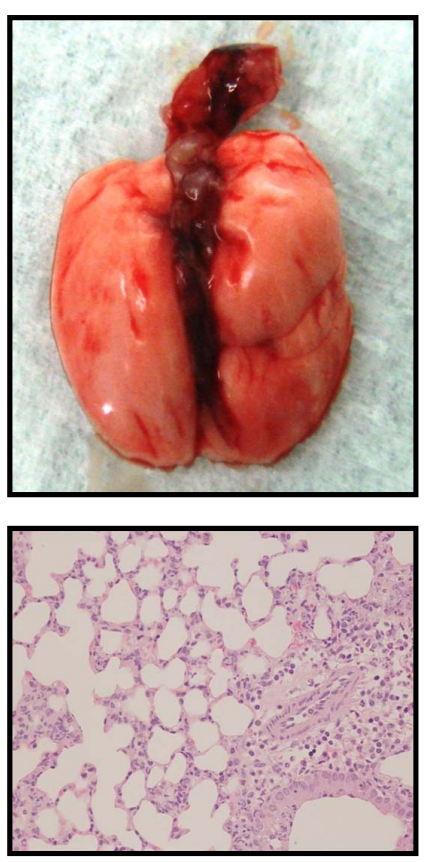

4 dpi
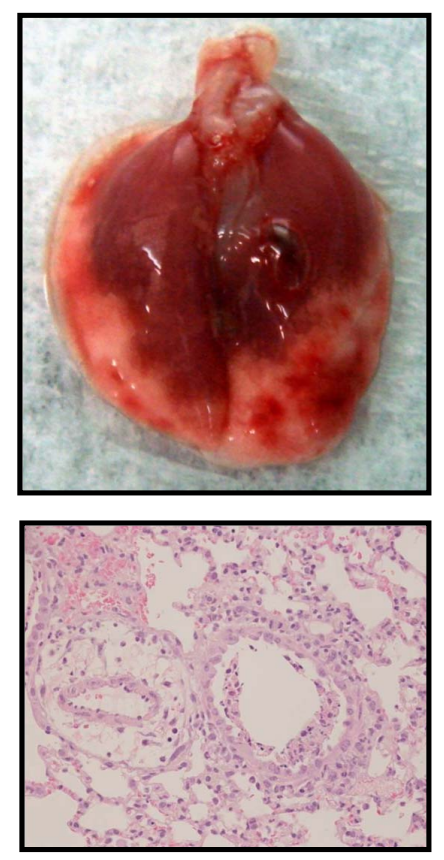

6 dpi
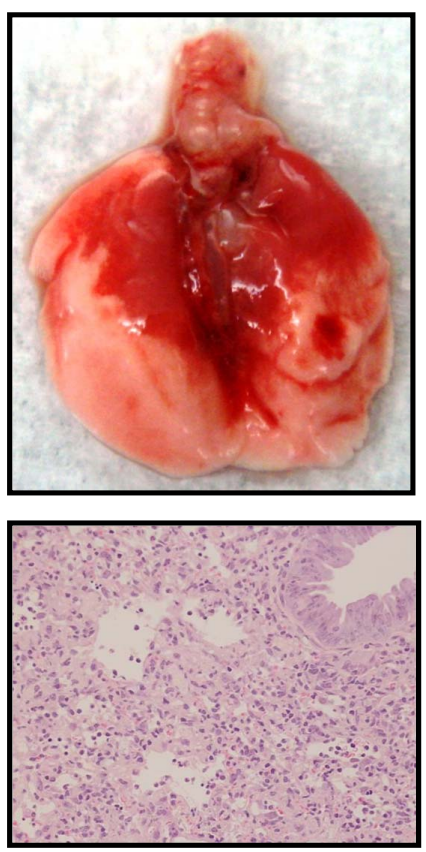

Fig. (1). HE-stained tissue sections of dissected lungs from infected BALB/c mice. Upper: Photos of dissected lungs with hemorrhagic pneumonia in BALB/c mice inoculated with $1000 \mathrm{FFUs}$ of mo-A/NC/20/99 at 2, 4 and 6 dpi. Lower: HE-stained tissue sections of the dissected lungs. 
(A)
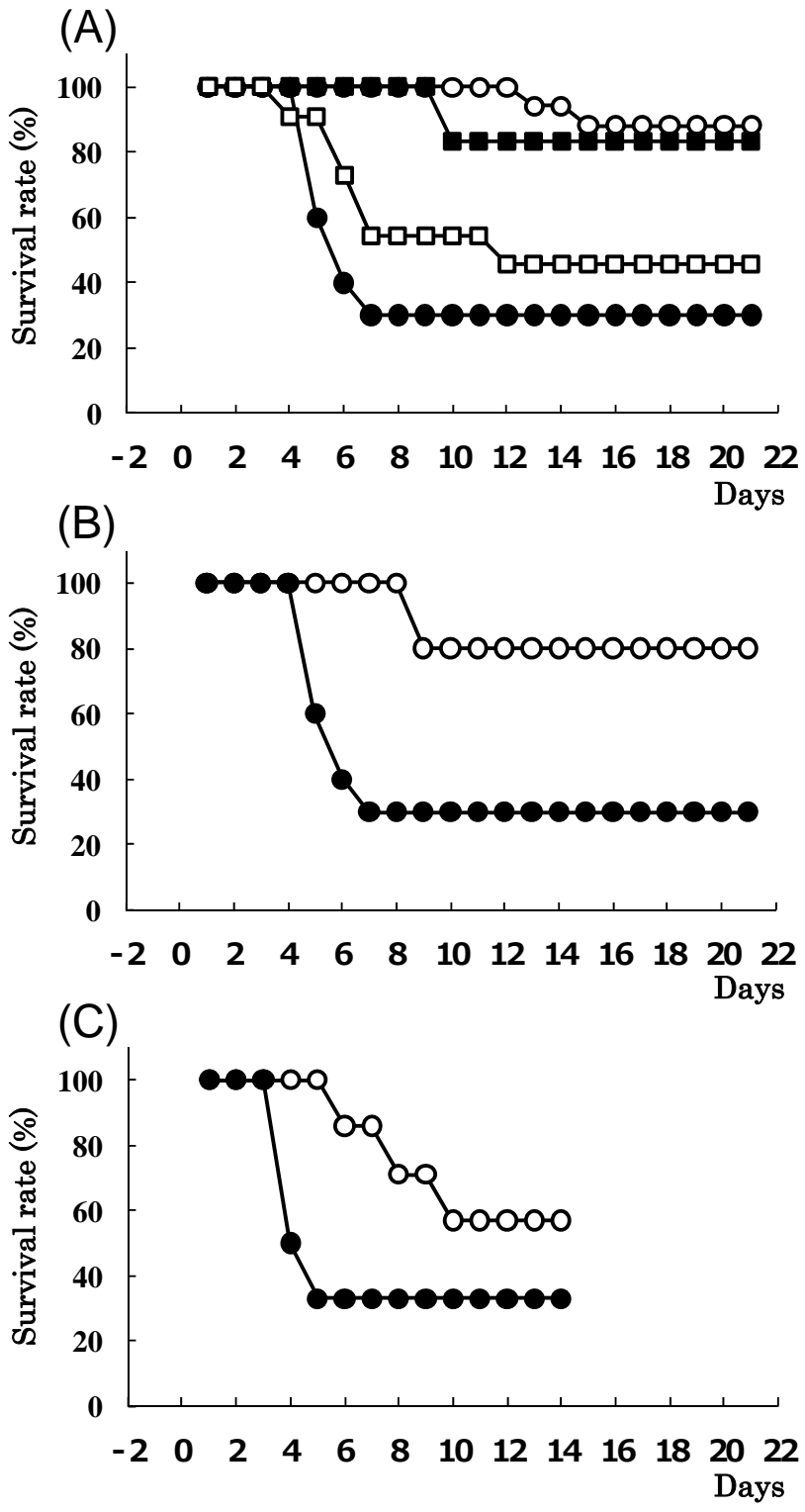

Fig. (2). Efficacy of IVIG against seasonal and pandemic H1N1 infections. A) To test the efficacy of IVIG after inoculation with seasonal H1N1, BALB/c mice inoculated with $1000 \mathrm{FFUs}$ of mo$\mathrm{A} / \mathrm{NC} / 20 / 99$ per head were left untreated (closed circle) or else treated with $5 \mathrm{mg}$ of IVIG (open circle) or a total of $2 \mathrm{mg}$ of Oseltamivir (closed square) per head at 48 hpi. In addition, the inoculated mice were treated with $5 \mathrm{mg}$ of IVIG per head at $72 \mathrm{hpi}$ (open square). B) To test the efficacy of IVIG against seasonal H1N1 before inoculation of the virus, BALB/c mice were left untreated (closed circle) or treated with $5 \mathrm{mg}$ of IVIG per head (open circle) at $48 \mathrm{hbi}$ with the same dose of H1N1 as above. C) To test the efficacy of IVIG against pandemic H1N1 after inoculation of the virus, BALB/c mice inoculated with 1000 FFUs of moA/Osaka/168/2009 per head were left untreated (closed circle) or treated with $5 \mathrm{mg}$ of IVIG per head (open circle) at $48 \mathrm{hpi}$.

effective, since the survival rate was significantly increased to $88 \%$, compared with $30 \%$ in the control. Interestingly, when mice were treated with Oseltamivir, a similar survival rate $(80 \%)$ was obtained. When mice were treated at $72 \mathrm{hpi}$,

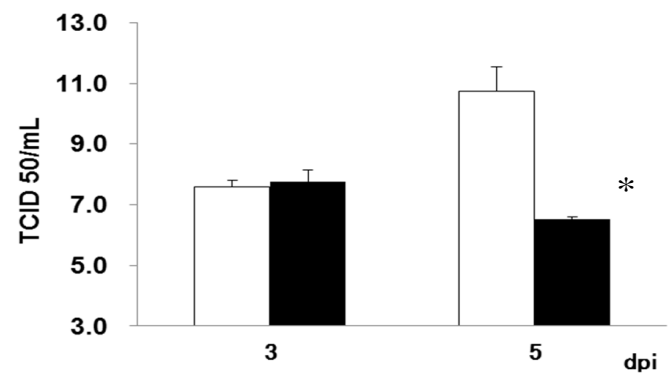

(B)

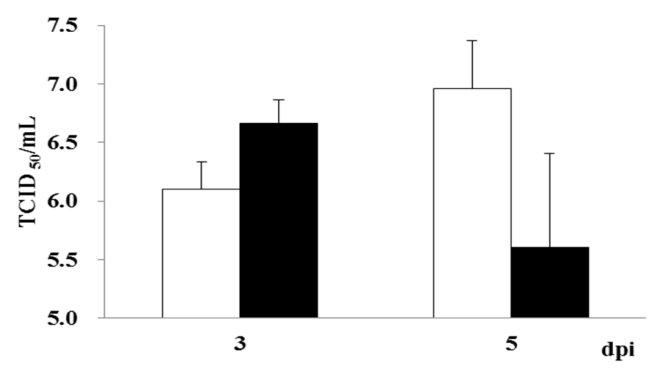

Fig. (3). Effect of IVIG treatment on viral propagation in the infected mouse lung. The amount of infectious virus in the lung from infected (closed bar) and control mice (open bar) at 3 and 5 days post inoculation (dpi) with seasonal H1N1 (A) and H1N1 pdm (B). The data showed the average and standard deviations of virus titer. Asterisk showed the statistical significance $\mathrm{P}<0.05$.

the survival rate greatly decreased to $45.5 \%$, suggesting the delayed treatment reduced by half the survival rate.

We also evaluated the preventive effect of IVIG against seasonal $\mathrm{H} 1 \mathrm{~N} 1$ infections in BALB/c mice. As shown in Fig. (2B), a similar survival rate, $80 \%$, was achieved with the intramuscular administration of IVIG $(5 \mathrm{mg} / \mathrm{head})$ at $48 \mathrm{hbi}$. Interestingly, severe clinical manifestations, such as respiratory symptoms and cyanosis, were not observed in all mice in the pretreated groups, suggesting that pre-treatment with IVIG may prevent the life-threatening influenza virus infection at least in mice. This suggestion was supported by a significant reduction of viral titers in the lungs from $\mathrm{BALB} / \mathrm{c}$ mice treated with IVIG at $48 \mathrm{hpi}: 10^{10.7}$ versus $10^{6.5} \mathrm{TCID}_{50} / \mathrm{ml}$ in control and IVIG-treated mice, respectively (Fig. 3A).

The treatment of BALB/c mice with IVIG increased the survival rate to $58 \%$ at 48 hpi with $\mathrm{H} 1 \mathrm{~N} 1 \mathrm{pdm}$, the survival rate being $33 \%$ for control mice (Fig. 2C). In fact, the experiments using SCID mice for $\mathrm{H} 1 \mathrm{~N} 1 \mathrm{pdm}$ revealed that the propagation of this virus also tended to decrease after IVIG treatment (Fig. 3B).

\section{DISCUSSION}

IVIG proved highly effective against influenza viral infections in mice, raising the survival rate from $30 \%$ to $88 \%$ for seasonal $\mathrm{H} 1 \mathrm{~N} 1$ infections and from $33 \%$ to $58 \%$ for H1N1 pdm infections. The treatment's efficacy depended on infection status, and was at least comparable with that 
of Oseltamivir treatment at $48 \mathrm{hpi}$. Interestingly, the administration of IVIG within 48 hpi significantly increased the survival rate of $\mathrm{H} 1 \mathrm{~N} 1$-infected individuals. This efficacy was also observed in the mice infected with $\mathrm{H} 1 \mathrm{~N} 1 \mathrm{pdm}$. The effect of IVIG differed between the seasonal and pandemic H1N1. The difference in survival rates could be due to different $\mathrm{HI}$ and VN titers of the IVIG against the viruses, i.e., 1:160 $\mathrm{HI}$ and 1:640 $\mathrm{VN}$ titers against seasonal $\mathrm{H} 1 \mathrm{~N} 1$ (A/NC/20/99) and 1:8 HI and 1:64 VN titers against H1N1 pdm (A/Osaka/168/2009) [3].

Immunoglobulin is believed to be a multi-functional plasma product [5]. There are several options against influenza-related diseases. Generally, IVIG has been used against complications such as influenza encephalopathy and the development of severe pneumonitis whereas Oseltamivir has been used against viral replication. Interestingly, the use of SCID mice revealed that IVIG reduced the viral titer, though only the 1:8 $\mathrm{HI}$ and 1:64 $\mathrm{VN}$ titers against $\mathrm{H} 1 \mathrm{~N} 1$ pdm. Thus, IVIG could be an option for treating serious influenza and the complications associated with it. In fact, in several cases, IVIG treatments have proven effective against influenza encephalopathy, severe pneumonitis and flu-related respiratory tract complications $[6,7]$. IVIG could be a secondline option for influenza with seasonal H1N1 and H1N1 pdm in patients with hematologic malignancies [8]. In addition, immunoglobulin $\mathrm{G}_{2}$ deficiency and bacterial co-infections seem to exacerbate H1N1 pdm infections [9-11].

Our study showed a significant effect of IVIG against the influenza virus in an animal model. The effectiveness of IVIG seems to be derived from Japanese blood donors who had received the seasonal influenza vaccines or had a history of natural infections with seasonal influenza viruses. Interestingly, the IVIG lot reactive with $\mathrm{H} 1 \mathrm{~N} 1 \mathrm{pdm}$ was detected even before the outbreak of H1N1 pdm [3]. The rate of donors who have antibodies cross-reactive with H1N1 pdm has increased in the population since the H1N1 pdm outbreak and the administration of trivalent inactivated influenza vaccine [12]. This finding suggests that vaccinations against the influenza virus generate cross-reacting antibodies to reassortant $\mathrm{H} 1 \mathrm{~N} 1 \mathrm{pdm}$ and may prevent lethal illness. Thus, the circulation of vaccinations may play an important role in producing cross-reactive immunoglobulin against new subtypes of the influenza virus. This study revealed the efficacy of IVIG for the prevention and treatment of both seasonal and pandemic H1N1 infections.

\section{CONFLICT OF INTEREST AND ACKNOWLEDGE- MENT}

This study was part of a collaborative project between Osaka University, Rakuno Gakuen University, The Research
Foundation for Microbial Diseases of Osaka University and Benesis Corporation. Research fund and human intravenous immunoglobulin (Venoglobulin- $\mathrm{IH}^{\circledR}$ ) used in this study were provided from Benesis Corporation. Tsujikawa M, Urayama $\mathrm{T}$ and Yunoki $\mathrm{M}$ are employee of Benesis Corporation. No ethical approval for human was required. Animal experiments were approved from the ethical committee for animal experiment of Rakuno Gakuen University.

We thank Dr. Yoshinobu Okuno, The Research Foundation for Microbial Diseases of Osaka University, and Mr. Fujio Kobayashi and Mr. Shoji Ideno, Benesis Corporation, for valuable discussions.

\section{REFERENCES}

[1] Study group appointed for influenza encephalopathy, Ministry of Health, Labour and Welfare of Japan. Guideline for influenza encephalopathy. 2009. [In Japanese]. Available at: http://www. mhlw.go.jp/kinkyu/kenkou/influenza/hourei/2009/09/dl/info092501.pdf

[2] The Japanese Respiratory Society. The JRS Guidelines for the Management of Community-Acquired Pneumonia in Adults. Nihon Rinsho 2003; 61(Suppl 2): 677-81. [In Japanese].

[3] Yunoki M, Kubota-Koketsu R, Urayama T, et al. Significant neutralizing activity of human immunoglobulin preparations against pandemic 2009 H1N1. Br J Haematol 2010; 148: 953-5.

[4] Hong DK, Tremoulet AH, Burns JC, Lewis DB. Cross-reactive neutralizing antibody against pandemic 2009 H1N1 influenza a virus in intravenous immunoglobulin preparations. Pediatr Infect Dis J 2011; 30: 67-9.

[5] Kazatchkine MD, Kaveri SV. Immunomodulation of autoimmne and inflammatory diseases with intravenous immune globulin. N Engl J Med 2001; 345: 747-55.

[6] Ogino Y, Takizawa D, Hayashi Y, et al. A case of influenza virusassociated encephalopathy successfully treated with a combination of mild hypothermia, anticytokine and anti virus. J Jpn Soc Intensive Care Med 2004; 11: 443-8. [In Japanese]

[7] Nakao T, Hamanishi T, Goto S, et al. Three case of influenza viral pneumonia. J Wakayama Med Soc 2001; 52: 405-10. [In Japanese]

[8] Casper C, Englund J, Boeckh M. How I treat influenza in patients with hematologic malignancies. Blood 2010; 115: 1331-42.

[9] Gordon CL, Johnson PDR, Permezel M, et al. Association between severe pandemic 2009 influenza A (H1N1) virus infection and immunoglobulin $\mathrm{G}_{2}$ subclass deficiency. Clin Infect Dis 2010; 50: 672-8.

[10] Palacios G, Hornig M, Cisterna D, et al. Streptococcus Pneumoniae coinfection is correlated with the severity of H1N1 pandemic influenza. Plos One 2010; 4: e8450.

[11] Murray RJ, Robinson JO, White JN, et al. Community-acquired Pneumonia due to pandemic A (H1N1) 2009 influenza virus and Methicillin resistant Staphylococcus aureus co-infection. Plos One 2010; 5: e8705.

[12] Katz J, Hancock K, Veguilla V, et al. Serum cross-reactive antibody response to a Novel Influenza A (H1N1) virus after vaccination with seasonal influenza vaccine. MMWR Morb Mortal Wkly Rep 2009; 58: 521-4. 